\title{
Estudio Radiográfico de la Asociación de Antioxidantes y Bifosfonatos en Remodelación Ósea Peri-Implantaria
}

\author{
Radiographic Antioxidants Association and Biphosphonates \\ Study in Bone Remodeling Peri-Implant
}

Escudero C.; Virga C.; Aguzzi A.; Hubert S. \& De Leonardi G.

ESCUDERO C.; VIRGA C.; AGUZZI A.; HUBERT S. \& DE LEONARDI G. Estudio Radiográfico de la asociación de antioxidantes y bifosfonatos en remodelación ósea peri-Implantaria. Int. J. Odontostomat., 10(3):531-537, 2016.

RESUMEN: Estudios previos han demostrado que los bisfosfonatos son potentes inhibidores de la resorción ósea. El aceite de oliva $(\mathrm{O})$ es rico en ácidos grasos monoinsaturados con potentes propiedades anti-oxidantes. El objetivo de este estudio fue estudiar el efecto del tratamiento de alendronato $(A L)$ y pamidronato $(P A)$ y de $O$ sobre la regeneración tisular. Las fórmulas se dosificaron $0,5 \mathrm{mg} / \mathrm{kg}$ de peso para $\mathrm{AL}$, y de $0,6 \mathrm{mg} / \mathrm{kg}$ de peso para PA. El O se administró en la dieta, $50 \mathrm{~g} / \mathrm{Kg}$. Cincuenta y cuatro ratas macho de la línea Wistar se dividieron en 6 grupos. El grupo control (C), recibió semanalmente $0,3 \mathrm{ml} / 100 \mathrm{~g}$ de peso corporal de solución salina vía subcutánea. El grupo $(\mathrm{AL})$ recibió semanalmente por vía subcutánea en el miembro posterior izquierdo. El grupo (PA) se colocó igual que el grupo anterior. El grupo (O) fue tratado en la alimentación y en las áreas de la cirugía recibieron inyección subcutánea con solución fisiológica. El grupo (ALO) recibió tratamiento combinado con $\mathrm{AL}$ y $\mathrm{O}$. El grupo (PAO) se trató igual al anterior. La cirugía consistió en una incisión longitudinal en las tibias realizando un defecto circular en la parte plana de cada tibia hasta llegar al hueso medular. Se tomaron radiografías a los $0,7,15,30,60$ y 90 días y fueron analizadas con el Software Image Pro Plus. Los estudios estadísticos se realizaron a través del análisis de la variancia a dos y tres criterios de clasificación. Se evidencio un incremento en la densidad mineral ósea promedio (DMO) conforme avanza el tiempo en todos los grupos, siendo evidentes con PA a los 60 días. El tratamiento O mostró eficacia en la remodelación ósea, observándose un pico a los 60 días. Esto sugiere que $\mathrm{O}$ representa una opción terapéutica para el tratamiento de las patologías óseas.

PALABRAS CLAVE: aceite de oliva, bisfosfonatos, remodelación ósea, implantes dentales.

\section{INTRODUCCIÓN}

Los bifosfonatos (BF) son fármacos análogos del pirofosfato, en los que un átomo de carbono sustituye al oxígeno entre los dos fosfatos variando sus cadenas laterales. Actúan provocando la apoptosis de los osteoclastos e inhibiendo así la resorción ósea, aunque el mecanismo exacto de acción es desconocido. (Aglias et al., 2007 \& Aramburú et al., 2008) También se ha demostrado que los BF inhiben la angiogénesis en ratas y en cultivos de células del cordón umbilical humano. Su acumulación en el hueso puede alcanzar una vida media de hasta 11 años. (Arambrú et al. \& Cicerale et al., 2010).

La eficacia biológica y clínica de los bifosfonatos varía según su tipo estructural. Generalmente, son dividi- dos en dos clases: los que contienen nitrógeno que, al mismo tiempo, son los más potentes, entre ellos: el alendronato, ibandronato, incadronato, olpadronato, pamidronato, risedronato y zoledronato; los que no poseen nitrógeno, que son, principalmente: clodronato, etidronato y tiludronato. (Corona et al., 2009 \& Cicerale et al.)

Los estudios realizados hasta el momento (incluyendo humanos, animales, in vivo e in vitro) han demostrado que los compuestos fenólicos del aceite de oliva tienen efectos positivos en varios biomarcadores fisiológicos, lo que implica que los compuestos fenólicos son parcialmente responsables por los beneficios para la salud asociados con la dieta del Mediterráneo.(Fritz et al., 2006 \& Guyton \& Hall, 2006). 
La biodisponibilidad de este compuesto se refiere al grado en el que se extrae de una matriz alimentaria y absorbido por el cuerpo (Hejdova et al., 2005 \& Guerra, 2009). La mayoría de las investigaciones sobre biodisponibilidad de aceite de oliva y los compuestos fenólicos se ha centrado en tres grandes grupos fenólicos: hidroxitirosol, tirosol y oleuropeína, en general, los compuestos fenólicos del aceite de oliva virgen demostraron que son fácilmente biodisponibles. (Miró-Casas et al., 2003 \& Impellizzeri et al., 2015)

En base a todo lo expuesto y a la bibliografía consultada, el presente trabajo pretende demostrar la efectividad clínica de AL y PA administrados por vía subcutánea en el sitio quirúrgico, y la eficacia de los suplementos de $\mathrm{O}$ tomando como parámetro la remodelación ósea, teniendo como hipótesis de la investigación que AL y PA administrados por vía subcutánea, inhiben la reabsorción ósea en animales de experimentación produciendo un sinergismo con la alimentación suplementada con los antioxidantes. Se pretende sumar estas drogas al arsenal terapéutico odontológico en el caso que fueran eficaces como neoformadores de hueso, con escasos efectos adversos pensando en una posterior evaluación clínica.

\section{MATERIAL Y METODO}

Preparación de las Formulaciones. Se prepararon soluciones de AL y PA para ser aplicados por vía subcutánea. La fórmula farmacéutica con $A L$ se prepararó con una dosificación de $0,5 \mathrm{mg} / \mathrm{Kg}$ de peso corporal, y la de PA con una dosificación de $0,6 \mathrm{mg} / \mathrm{Kg}$ de peso corporal. El O se administró junto con la dieta, 50 g/Kg de comida.

Preparación de los Animales de Experimentación. Cincuenta y cuatro ratas macho de la línea Wistar de peso $160 \pm 20 \mathrm{~g}$, se dividieron en 6 grupos de 9 ratas cada uno. Un grupo actuó como grupo control (C). Los animales de este grupo recibieron semanalmente 0,3 $\mathrm{ml} / 100 \mathrm{~g}$ de peso corporal de solución salina por vía subcutánea cercana a la intervención quirúrgica y se administró agua corriente de red como agua de bebida. El segundo grupo $(A L)$ se dosificó semanalmente 0,5 $\mathrm{mg}$ de $\mathrm{AL} / \mathrm{Kg}$ de peso corporal por vía subcutánea profunda en el miembro posterior izquierdo cercano a la zona quirúrgica, y en el miembro posterior derecho se le aplicó solución salina como el grupo control. El tercer grupo (PA) recibió semanalmente $0,6 \mathrm{mg}$ de $\mathrm{PA} / \mathrm{Kg}$ de peso corporal por vía subcutánea profunda en el miembro posterior izquierdo cercano a la zona quirúrgica, y en el miembro posterior derecho se suministró solución salina como el grupo control. El cuarto grupo (O), recibirá tratamiento con $\mathrm{O}$, siendo alimentados ad libitum, con una ración de $40 \mathrm{~g}$ día/rata, al que se le adicionó previamente el principio activo durante el tiempo que duró el experimento y en las áreas de las cirugías recibieron inyección subcutánea de solución salina como el grupo control. El quinto grupo de tratamiento se administró en forma combinada AL subcutáneo y ADO por vía oral en la dieta $(A L+A D O)$. El sexto grupo de tratamiento combinado PA subcutáneo y ADO por vía oral en la dieta (PA+ADO)

Los animales se mantuvieron en bioterio, en jaulas colectivas, con alimento balanceado y agua de bebida ad libitum, a una temperatura de $22-26^{\circ} \mathrm{C}$, con un ciclo luz-oscuridad: $12 \mathrm{~h}$-12 $\mathrm{h}$ durante el tiempo que duró el experimento. El manejo de los animales es realizado siguiendo los lineamientos del Nacional Institute of Health $(\mathrm{NIH})$ para el uso y cuidado de animales de experimentación.

Al inicio del experimento los animales fueron anestesiados con una solución de ketamina/xilazina en relación $8 \mathrm{mg} / 1,28 \mathrm{mg}$ respectivamente por cada $100 \mathrm{~g}$ de peso corporal. Previa asepsia del campo quirúrgico con yodopovidona. Se realizó con bisturí Bard Parker y hoja N ${ }^{\circ} 15$ una incisión longitudinal en ambas tibias y se procedió al decolado hasta llegar a exponer el hueso. Con una fresa número 6 y a rotación manual para no producir necrosis ósea, se realizó una cavidad en la parte plana de cada tibia hasta llegar al hueso medular. Dicha cavidad no fue rellenada con ningún material y sólo será reparada por su propio coágulo. Luego de realizada las intervención quirúrgica, se recolocaron los planos en posición y se suturó la herida con hilo reabsorbible.

Los animales fueron tratados según normas universales de asepsia. Al finalizar el experimento se sometieron a eutanasia los animales mediante inyección intracardíaca de cloruro de potasio, bajo anestesia general. (Puel et al., 2008)

Se efectuaron determinaciones en los días $0,7,15,30$, 60, 90 administrando la solución salina, el AL y el PA cada 7 días; y en forma continua el aceite de oliva al cuarto, quinto y sexto grupo.

Los sacrificios para la toma de muestras fueron a los $0,7,15,30,60$ y 90 días. En todos los tiempos experimentales, incluidos se realizaron tomas radiografías de las tibias. 
Estudios Radiográficos. Se tomaron radiografías de ambas tibias, inmediatamente posterior a la cirugía y en cada uno de los tiempos experimentales, por técnica de cono corto con tiempo de exposición de 0,4 s. Las muestras fueron analizadas con el Software Image ProPlus versión 4,1 de Media Cibernetics, diseñado para trabajar con microscopios confocales Olympus. Este software mide densidad óptica (escala de grises) obteniendo valores numéricos que son registros de las siguientes zonas: hueso medular, hueso cortical, zona problema y área circundante a la zona problema. Dicha densidad de los diferentes sectores será cuantificado $(\mathrm{mg} \mathrm{Ca}+2 / \mathrm{cm} \Sigma)$ utilizando un patrón de aluminio.

Estadística. La comparación de los datos se realizó por análisis de la varianza a dos criterios de clasificación (tratamientos: $C, A L, P A, A D O, A L+A D O$, PA+ADO, y tiempos de tratamiento: 3060 y 90 días). Se utilizó tests paramétricos o no en función que los resultados obtenidos permitan o no verificar distribución normal de las variables utilizadas (Test de Kolmorow Smirnov). Se consideraron diferencias significativas si $p>0,05$. Este estudio estadístico planteado además permitió determinar si existe asociación entre los efectos de los tratamientos y el tiempo. Para el análisis de datos, construcción de gráficos y análisis estadísticos se utilizó el software (GraphPad Prism 2.0. GraphPad Software, San Diego, CA USA).

\section{RESULTADOS}

Se obtuvieron radiografías de ambas tibias en todos los grupos y en todos los tiempos. Se obtuvieron 5 medidas de DO de la perforación y 5 medidas de DO de la zona de hueso sano próxima a la perforación, en cada tibia. Se promediaron las DO de cada zona y se obtuvo la diferencia entre ellas. (DO perforación - DO hueso sano).

Análisis estadístico en estudios radiográficos. A continuación se presentan, las medidas descriptivas para las diferencias de Densidad Óptica (DO) existente entre la zona de perforación y el entorno próximo, considerando las tibias, según el tiempo de toma de las muestras. (Fig. 1).

Cabe aclarar que a consecuencia del modelo experimental empleado, la cantidad de valores medidos va disminuyendo a medida que se avanza en la escala temporal.

Para estudiar los efectos producidos por los dos factores considerados en el experimento: etapa y tratamiento, se utilizó un modelo factorial de tratamientos. El efecto simultáneo de ambos factores se denomina interacción.

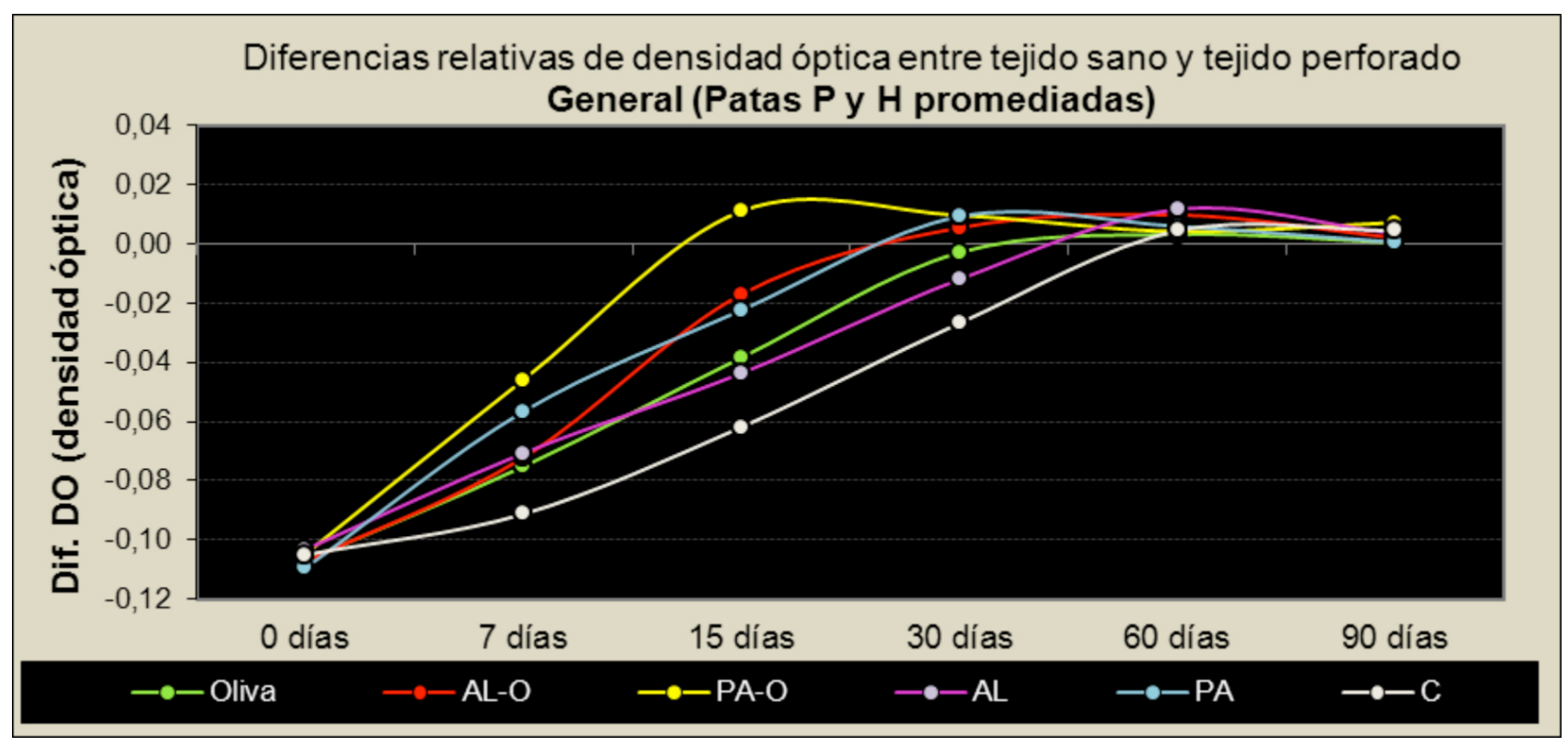

Fig.1. Media para las diferencias de la variable DO. General. 


\section{DISCUSION}

El objetivo de esta investigación fue evaluar el efecto de dos bisfosfonatos, Al y PA, administrados en el modelo experimental descrito y $\mathrm{O}$ incorporado en la dieta de los animales y las respectivas combinaciones para observar la remodelación ósea en cavidades neoformadas a través del análisis de distintas variables como radiográfico, FA, histología e histomorfometría y ensayos biomecánicos.

El aumento de peso de todos los animales fue semejante al final del experimento, esto demuestra un control de la alimentación (libre demanda) y la respuesta del animal a la cirugía; durante cirugías invasivas el peso del animal puede disminuir y las con- diciones generales de salud pueden verse afectadas, derivando en un retraso en la recuperación de los animales.

Estudios previos demostraron que la concentración de AL afecta el porcentaje de captación de rojo neutro (ANOVA $p<0,01$ ). No se observó diferencia en la captación hasta $10 \mu \mathrm{g} / \mathrm{ml}$ aunque para concentraciones de $20 \mu \mathrm{g} / \mathrm{ml}$ o mayores se observó un descenso significativo (HSD Tukey $p<0,01)$. Se pudo determinar entonces dos rangos de concentración: Uno $(0$ a $10 \mu \mathrm{g} / \mathrm{ml}$ ) en el que la viabilidad celular no es afectada y otro (10 a $20 \mu \mathrm{g} / \mathrm{ml})$ donde comienza a afectarse dicha viabilidad. Esta variable permitió dosificar la droga a utilizar, de manera óptima para no producir toxicidad en los ensayos en animales.

Tabla I. Cuadro de ANOVA para las diferencias (Perforación - Entorno) de la variable DO.

\begin{tabular}{lccccc}
\hline \multicolumn{4}{l}{ Pruebas de los efectos inter-sujetos. } & \multicolumn{4}{c}{ Variable dependiente: Diferencia de DO } \\
\hline Fuente & SC & gl & MC & F & p-valor \\
P_H & .001 & 1 & .001 & 1.440 & 0.232 \\
Droga & .015 & 5 & .003 & 6.744 & $\mathrm{p}<0.001$ \\
Etapa & .439 & 5 & .088 & 198.560 & $\mathrm{p}<0.001$ \\
Droga * Etapa & .022 & 25 & .001 & 2.027 & 0.004 \\
\hline
\end{tabular}

SC (suma de cuadrados); gl (grados de libertad); MC (media cuadrática); F (relación de factores). (a) $\mathrm{R}$ cuadrado $=.867$ ( $\mathrm{R} 2$ corregida $=.814)$.

Tabla II. DHS de Tukey. Subconjuntos homogéneos según tratamiento o droga.

\begin{tabular}{llccc}
\hline Droga & $\mathrm{n}$ & \multicolumn{3}{c}{ Subconjunto } \\
& & 1 & 2 & 3 \\
\hline Control & 45 & -0.0553 & & \\
AL & 40 & -0.0475 & -0.0475 & \\
Oliva & 41 & -0.0461 & -0.0461 & \\
PA & 38 & & -0.0432 & \\
ALO & 44 & & -0.0397 & \\
PAO & 40 & & & -0.0258 \\
Significación & & 0.102 & 0.553 & 1.000 \\
\hline
\end{tabular}

Tabla III. DHS de Tukey. Subconjuntos homogéneos según etapa.

\begin{tabular}{lrrrrr}
\hline Etapa & $\mathrm{n}$ & \multicolumn{4}{c}{ Subconjunto } \\
& 1 & 1 & 2 & 3 & 4 \\
\hline 0 días & 53 & -0.1060 & & & \\
7 días & 56 & & -0.0720 & \\
15 días & 47 & & & -0.0273 & \\
30 días & 36 & & & & -0.0028 \\
60 días & 23 & & & 0.0035 \\
90 días & 33 & & & & 0.0076 \\
Significa ción & & 1.000 & 1.000 & 1.000 & 0.253 \\
\hline
\end{tabular}


En este trabajo, el análisis de la DMO permitió establecer la calidad de hueso formado en el defecto logrado quirúrgicamente, a través de un aumento de la radiopacidad de esta zona, conforme al avance del tiempo.

\section{Esta investigación mostró en cuanto a la va- riable estudio radiográfico diferencias estadísticamente significativas $(p<0,001)$ respecto al factor droga empleada y al factor etapa. Por otro lado, la interacción Droga * Etapa resultó significativa, in- dicando que las diferencias entre tratamientos de- penden de la etapa considerada. Las mayores dife- rencias se registraron a los 7 y 15 días.}

De acuerdo al resultado de la prueba de ANOVA, la variable pata problema o pata homóloga, no fue un factor determinante, tal como lo expresa el estadístico calculado ( $p=0,232)$, lo que estaría indicando que los bisfosfonatos colocados en forma subcutánea en el sitio quirúrgico poseen un efecto sistémico evidenciándose efectividad terapéutica en la muestra pata contralateral.

Al analizar las diferencias entre etapas de tratamiento, se observa que pasados los 30 días, las variaciones son mucho menos acentuadas, evidenciando que el proceso se produce principalmente en el rango de 0 a 30 días.

Los grupos C, AL y Oliva mostraron un comportamiento semejante en general, pero los grupos Oliva y $\mathrm{AL}$, llegaron a niveles aceptables antes que el grupo control. El tratamiento $O$ evidenció eficacia terapéutica en la remodelación ósea, aún siendo administrado por vía sistémica en la dieta observándose un pico máximo a los 60 días de tratamiento y luego una meseta hasta los 90 días.

Los grupos Oliva, AL-O PA y AL, pueden agruparse en otro subconjunto homogéneo, en un rango central y excluyendo los extremos: el grupo PA-O, (de rápida respuesta) y el grupo $\mathrm{C}$, presentó valores por debajo de todos los grupos experimentales.

El análisis radiográfico es una importante herramienta de adyuvante para la evaluación clínica, ya que es un método rápido, no invasiva y de bajo costo y puede producir resultados similares a los de los estudios histológicos. La medición de la DMO por densitometría radiográfica se ha realizado en un número de estudios, y su eficacia se ha confirmado.
Hejdova et al., analizaron la DMO en 50 mujeres posmenopáusicas con osteoporosis durante su primer año de tratamiento., después de la administración sistémica de AL, utilizando dosis de $1 \mathrm{mg} / \mathrm{kg}$ por semana, pudiendo demostrar que la DMO lumbar aumentó significativamente en un $7,0 \%(P<0,001)$, la DMO del cuello del fémur en un $4,3 \%(P<0,01)$.

Esta tesis pudo demostrar un aumento de la DMO en forma significativa, aun utilizando dosis menores a $1 \mathrm{mg} / \mathrm{kg}$ de $\mathrm{AL}$, lo cual disminuiría potencialmente la aparición de efectos adversos.

Impellizzeri et al., confirmaron con sus trabajos que la oleuropeína aglicona ejerce efectos beneficiosos en el modelo experimental de artritis inducida por colágeno, también fue evaluado su efecto en un régimen terapéutico de post-tratamiento $(40 \mathrm{mg} / \mathrm{kg}$ ), comenzando el tratamiento al día 28. Oleuropeína administrada post-tratamiento en ratones también mostró una reducción en el desarrollo de la artritis y una artritis significativamente menor puntuación de comparación con los de control.

Miró-Casas et al., demostraron en su trabajo la biodisponibilidad en humanos de un compuesto fenólico presentes en el aceite de oliva consumido en su forma natural. Se encontró que el tirosol fue absorbido a partir de aceite de oliva virgen administrada y que una parte de la dosis se excreta en las próximas 24 horas. Este estudio también muestra la viabilidad de los estudios de biodisponibilidad controladas de compuestos fenólicos a partir de fuentes de la dieta en los seres humanos. Estos trabajos soportan los resultados de esta investigación al poder analizar los resultados obtenidos en los animales de experimentación.

\section{CONCLUSION}

En base a esta investigación se considera al O de acción dual capaz de disociar el remodelado óseo reequilibrándolo hacia la formación de hueso, actúa a nivel de todos y cada uno de los determinantes de la resistencia ósea, incrementando de forma significativa y precoz la $\mathrm{DMO}$, mejorando cualitativamente la estructura del hueso trabecular y cortical y preservando la mineralización, el tamaño y la estructura de los cristales minerales incluso a dosis elevadas. De esta manera, O mejora significativamente las propiedades biomecánicas del hueso. 
En cuanto a la variable radiografía se puede observar un incremento en la DMO conforme avanza el tiempo de todos los grupos problemas con respecto al control, siendo más evidentes los tratamientos con $\mathrm{PA}$ a los 60 días.

El tratamiento $\mathrm{O}$ evidenció eficacia terapéutica en la remodelación ósea, aún siendo administrado por vía sistémica en la dieta observándose un pico máximo a los 60 días de tratamiento

ESCUDERO, C.; VIRGA, C.; AGUZZI, A.; HUBERT, S. \& DE LEONARDI, G. Radiographic antioxidants association and biphosphonates study in bone remodeling peri-implant. Int. J. Odontostomat., 10(3):531-537, 2016.

ABSTRACT: Previous studies have shown that bisphosphonates are potent inhibitors of bone resorption. Olive oil $(O)$ is rich in monounsaturated fatty acids with potent anti-oxidant properties. The objective of this work was to study the effect of alendronate treatment $(A L)$ and pamidronate $(P A)$ and $O$ on tissue regeneration. Formulas $0.5 \mathrm{mg} / \mathrm{kg}$ for AL dosed, and $0.6 \mathrm{mg} / \mathrm{kg}$ for PA. O was administered in the diet, $50 \mathrm{~g} / \mathrm{kg}$. Fifty-four male rats Wistar were divided into 6 groups. The control group (C) received weekly $0.3 \mathrm{ml} / 100 \mathrm{~g}$ body weight of saline subcutaneously. The group (AL) received a weekly dose subcutaneously in the left posterior limb. The group (PA) was placed as the previous group. The group $(\mathrm{O})$ was treated in food and in the areas of surgery received subcutaneousinjection with saline. The group (ALO) received combined treatment with $\mathrm{Al}$ and $\mathrm{O}$. The group (PAO) was treated the same as before. Surgery consisted of a longitudinal incision in the warm using a circular on the flat side of each tibia until the medullary bone defect. X-rays at $0,7,15,30,60$ and 90 days were taken and analyzed with Image Pro Plus Software. Statistical studies were conducted through analysis of variance to two and three classification criteria. Results: an increase in the average bone mineral density (BMD) was evident as time progresses in all groups, with PA still evident at 60 days. Or treatment showed efficacy in bone remodeling observed a peak at 60 days. Conclusions: This suggests that $O$ represents a therapeutic option for the treatment of bone disease.

KEY WORDS: olive oil, bisphosphonates, bone remodeling, dental implants.

\section{REFERENCIAS BIBLIOGRÁFICAS}

Agalias, A.; Magiatis, P.; Skaltsounis, A. L.; Mikros, E.; Tsarbopoulos, A.; Gikas, E.; Spanos, I.; Manios, T. Anew process for the management of olive oil mill waste water and recovery of natural antioxidants. J. Agric. Food Chem., 55:2671-2676, 2007.
Aramburú, G.; Aguzzi, A.; Ricco, V.; Hubert, \& Virga, C. Study of a Subcutaneous Formulation on the Basis of Alendronato. J. Den. Res., 84:13-15, 2005.

Aramburú, G.; Aguzzi, A.; Ricco, V.; Hubert, S.; Aguilar, J.; Konigheim, B. \& Virga, C. Estudio de una formulación subcutánea en base a Alendronato. Acta odontológica Venezolana, 46(4):5-9, 2008.

Aramburú, G.; Aguzzi, A. \& Virga, C. Estudio histopatológico sobre el efecto de una nueva formulación de alendronato administrado por vía subcutánea. Acta Odontológica Venezolana, 47(3):17-21, 2009.

Cicerale, S.; Lucas, L. \& Keast, R. Biological activities of phenolic compounds present in virgin olive oil. Int. J. Mol. Sci., 11(2):458-79, 2010.

Corona, G.; Spencer, J. P. E. \& Dessi, M. A. Extra virgin olive oil phenolics: absorption, metabolism, and biological activities in the GI tract. Toxical. Ind. Health, 25:285-293, 2009.

Ebetino, F. H.; Hogan, A. M.; Sun, S.; Tsoumpra, M.K.; Duan, X.; Triffitt, J. T. et.al. The relationship between the chemistry and biological activity of the bisphosphonates. Bone, 49(1):20-33, 2011.

Enjuanes, A.; Ruiz-Gaspà, S.; Peris, P.; Ozalla, D.; Alvarez, L.; Combalia, A. et al. The effect of the alendronate on OPG/RANKL system in differentiated primary human osteoblasts. Endocrine, 37(2):322-8, 2010.

Fritz, D. T.; Jiang, S.; Xu, J. \& Rogers, M. B. A polymorphism in a conserved posttranscriptional regulatory motif alters bone morphogenetic protein 2 (BMP2) RNA:protein interactions. Mol. Endocrinol, 20(7):1574-86, 2006.

Ganong, W. Control hormonal, metabolismo del calcio y fisiología del hueso. En: Medina Soriano. Fisiología Médica. 20 ed. México DF. Editorial El manual moderno. 2006.

Guerra, M. Mediterranean diet in a healthy perspective of the richness of functional foods. Hig. Aliment, 23(170/ 171):22-27, 2009.

Guyton, A. C. \& Hall, J. Endocrinología y reproducción. En: Fisiología Médica 11 ed. España. Editorial Elsevier; 2006.

Hejdova, M.; Palicka, V.; Kucera, Z. \& VIcek, J. Effects of alendronate and calcitonin on bone mineral density in postmenopausal osteoporotic women. An observational study. Pharm. World Sci., 27(3):149-53, 2005.

Impellizzeri, D.; Talero, E.; Siracusa, R.; Alcaide, A.; Cordaro, M. Zubelia, J. et al. Protective effect of polyphenols in an 
ESCUDERO C.; VIRGA C.; AGUZZI A.; HUBERT S. \& DE LEONARDI G. Estudio Radiográfico de la asociación de antioxidantes y bifosfonatos en remodelación ósea periImplantaria. Int. J. Odontostomat., 10(3):531-537, 2016.

inflammatory process associated with experimental pulmonary fibrosis in mice. Br. J. Nutr., 114(6):853-65, 2015.

Madrona, A.; Pereira-Caro, G.; Mateos, R.; Rodríguez, G.; Trujillo, M.; Fernández-Bolaños, J. \& Espartero, J. Synthesis of Hydroxytyrosyl Alkyl Ethers from Olive Oil Waste Waters. Molecules, 14:1762-1772, 2009.

Miró-Casas, E.; Covas, M. I.; Fitó, M.; Farré-Albadalejo, M.; Marrugat, J. \& de la Torre, R. Tyrosol and hydroxytyrosol are absorbed from moderate and sustained doses of virgin olive oil in humans. Eur. J. Clin. Nutr., 57(1):18690, 2003.

NHI Guide for the care and use for Laboratory Animals.us. Departament of Health and Human Service, public health service, National Institutes of Health publication 1985. nro.85.23

Puel, C.; Mardon, J.; Agalias, A.; Davicco, M. J.; Lebecque, P.; Mazur, A. et.al. Major phenolic compounds in olive oil modulate bone loss in an ovariectomy/inflammation experimental model. J. Agric. Food. Chem., 56(20):941722, 2008.

Reynaga-Montecinos, B. \& Zeni, S. Marcadores bioquímicos del remodelamiento óseo. Utilidad clínica. Acta Bioquímica Clínica Latinoamericana, 43(2):27-32, 2009.

Rigalli, A. \& Di Loreto, V. Experimental surgical models in the Laboratory Rat. Taylor and Francis Group. CRC Press. Boca Ratón. USA. 2009.

Ross, M.; Kaye, G. \& Pawlina, W. Tejido Óseo. En: Histología, Texto y Atlas color con Biología celular y molecular. 4ta.ed. Madrid, España. Editorial Médica Panamericana, 2005.

Russell, R. G. Bisphosphonates: The first 40 years. Bone, 49(1):2-19, 2011.

Tripoli, E.; Giammanco, M.; Tabacchi, G.; Di Majo, D.; Giammanco, S. \& La Guardia, M. The phenolic compounds of olive oil: structure, biological activity and beneficial effects on human health. Nutr. Res. Rev., 18:98-112, 2005.

Tsarbopoulos, A.; Gikas, E.; Papadopoulos, N.; Aligiannis, N. \& Kafatos, A. Simultaneous determination of oleuropein and its metabolites in plasma by high-performance liquid chromatography. J. Chromatogr. B. Analyt. Technol. Biomedical chromatografy BMC, 24(5):506-515, 2010.

Waterman, E. \& Lockwood, B. Active components and clinical applications of olive oil. Altern. Med. Rev., 12(4):331-42, 2007.

\author{
Dirección para Correspondencia \\ Dra. Alejandra Aguzzi \\ Profesora Titular \\ Cátedra de Farmacología y Terapéutica A \\ Facultad de Odontología \\ Universidad Nacional de Córdoba \\ ARGENTINA
}

Email: aleceagu@yahoo.com.ar

Recibido : 13-07-2016

Aceptado: 17-10-2016 